\title{
Molecular analysis of Fanconi anemia and mismatch repair genes in patients with colorectal carcinoma
}

\author{
MARIA COLOMBINO ${ }^{1,5}$, ANTONIO AVALLONE ${ }^{2}$, FRANCESCO IZZO², FABIANA TATANGELO ${ }^{2}$, \\ MARIO BUDRONI ${ }^{3}$, ANTONIO COSSU ${ }^{4}$, FRANCESCO GALIMI ${ }^{5}$, PASQUALE COMELLA ${ }^{2}$, GERARDO BOTTI ${ }^{2}$, \\ MARIA CRISTINA SINI ${ }^{1}$, FRANCESCO TANDA ${ }^{4}$ and GIUSEPPE PALMIERI ${ }^{1}$ on behalf of the \\ Southern Italy Cooperative Oncology Group (SICOG) \\ ${ }^{1}$ Istituto di Chimica Biomolecolare-C.N.R., Sassari; ${ }^{2}$ Istituto Nazionale Tumori Fondazione Pascale, Napoli; \\ ${ }^{3}$ Servizio di Epidemiologia, Azienda Unità Sanitaria Locale 1, Sassari; ${ }^{4}$ Servizio di Anatomia Patologica, Azienda \\ Ospedaliero Universitaria, Sassari; ${ }^{5}$ Dipartimento di Scienze Biomediche/INBB, Università di Sassari, Sassari, Italy
}

Received July 27, 2010; Accepted October 5, 2010

DOI: $10.3892 /$ or.2011.1169

\begin{abstract}
A cohort of 206 consecutively-collected patients with colorectal carcinoma (CRC) were screened for germline mutations in the principal DNA mismatch repair (MMR) genes, $\mathrm{MLH1}$ and $\mathrm{MSH} 2$, and in the Fanconi anemia (FA) genes involved in homologous recombination DNA repair. Mutation analysis was performed by denaturing high-performance liquid chromatography (DHPLC) and automated sequencing. Available paraffin-embedded tumor tissues were evaluated for gene expression by immunohistochemistry. Genes of the FA pathway were found to participate in CRC pathogenesis, being silenced during disease progression and metastasis formation. Conversely, $M L H 1$ and $M S H 2$ genes seem to be inactivated at earlier stages of the disease. Finally, very few (about 5\%) cases presented a simultaneous inactivation of the MMR and FA genes. Overall, our findings indicated that: i) mismatch DNA repair remains the main mechanism to be altered at both germline and somatic levels among CRC patients; ii) functional impairments of mismatch DNA repair and FA-related repair may represent two different pathogenetic alterations which are concurring in colorectal cancer progression.
\end{abstract}

\section{Introduction}

Colorectal carcinoma (CRC) represents the second most frequently occurring cancer in both sexes among Western

Correspondence to: Dr Giuseppe Palmieri, Istituto di Chimica Biomolecolare-Sezione di Sassari, C.N.R., Traversa La Crucca, 3 Baldinca Li Punti, 07100 Sassari, Italy

E-mail: gpalmieri@yahoo.com

Abbreviations: CRC, colorectal carcinoma; DHPLC, denaturing high-performance liquid chromatography; FA, Fanconi anemia; MMR, mismatch DNA repair; PCR, polymerase chain reaction

Key words: colorectal tumorigenesis, DNA repair, immunohistochemistry, mutation analysis countries (1). In Italy, approximately 30,000 new cases of colorectal cancer are diagnosed every year (corresponding to about 70 new cases per year per 100,000 inhabitants) (1).

Malignant transformation of the colorectal epithelium is thought to occur through sequential accumulation of genetic alterations (2). Genomic instability, which is mostly due to a defective replication fidelity, has been widely recognized as one of the main pathogenetic factors involved in colorectal tumorigenesis (3); it has been demonstrated that not only epithelial but also stromal genetic instability possibly contributes to development of colorectal carcinoma (4). Tumors with nonfunctional DNA mismatch repair (MMR) display a genomic instability as inferred by detection of ubiquitous somatic variation in length of microsatellite sequences (5). Genetic instability has been associated with the presence of mutations or inactivation of the two principal MMR genes, $M L H 1$ and $\mathrm{MSH} 2$ (though the most prevalently alterated is $\mathrm{MLH1}$ ) $(5,6)$. Patients with defective MMR mechanisms have more probabilities to present a familial recurrence of colorectal carcinoma, frequently underlying the occurrence of a hereditary non-polyposis colorectal carcinoma (HNPCC) syndrome $(7,8)$. Mutations of the MLH1/MSH2 genes have been documented at various rates in different populations, ranging from approximately $25 \%$ to more than $90 \%$ of CRC families $(9,10)$. Conversely, inactivation of the MMR system has been documented at lower frequencies (nearly 20\%) of the sporadic colorectal cancers (11); majority of them is associated with MLH1 promoter hypermethylation (12), with $10-20 \%$ cases carrying an MLH1/MSH2 mutation $(11,13)$. Therefore, additional genes and pathways may contribute to induce genomic instability during colorectal tumorigenesis.

Recently, proteins encoded by genes involved into the pathogenesis of the Fanconi anemia (FA), a rare pediatric disorder of chromosome instability characterized by a high incidence of leukemias and solid tumors (14), have been demonstrated to cooperate in the recognition and repair of damaged DNA (15). To date, 13 genes have been demonstrated to participate to pathogenesis of Fanconi's anemia, and 1 of them is identical to the well-known breast cancersusceptibility gene, BRCA2 (Fanc-D1) (15). Studies dissecting the molecular interactions of FA gene products have indicated 
that five of them (Fanc-A, Fanc-C, Fanc-E, Fanc-F, and Fanc-G) form a nuclear multimeric complex which is required for the activation of the downstream events in DNA repair $(15,16)$. Nevertheless, the functional classification of $B R C A 2$ as a FA gene as well as the discovery that Fanc-D2 and BRCA1 proteins indeed interact (17-19) strongly support the hypothesis that the FA pathway is involved in cancer susceptibility through regulation of the homologous recombination DNA repair mechanisms. Moreover, these findings suggest that impairment of the FA pathway may have an impact on sensitivity of tumors to conventional chemotherapy with DNA cross-linking agents (alkylating anticancer drugs, such as cisplatin or platinum analogues) (20) or to inhibitors of DNA repair $(21,22)$.

The purpose of this study was to evaluate whether the FA genes forming the above-mentioned nuclear multimeric complex (Fanc-A, Fanc-C, Fanc-E, Fanc-F, and Fanc-G) might be altered in advanced colorectal cancer, which is now routinely treated with oxaliplatin-based combination chemotherapy (oxaliplatin is a relatively new platinum analogue) (23). Furthermore, we here assessed the occurrence of any relationship between alterations in FA and $M L H 1 / M S H 2$ genes during colorectal tumorigenesis and progression.

\section{Materials and methods}

Patients and controls. Patients with histologically-proven diagnosis of colorectal carcinoma (CRC) were recruited from clinics at the University of Sassari and National Cancer Institute of Naples. To avoid any bias, CRC patients were consecutively collected from March 2006 to June 2008; they were included regardless of age at diagnosis and disease features. Family history for cancer was evaluated through specific questionnaires during the follow-up visits at the different participating institutions. Patients were classified as familial cases when at least three family members (including the proband) among first- and second-degree relatives were affected by colorectal carcinoma; remaining patients who did not match these criteria were classified as sporadic cases. All information were verified through careful analysis of the hospital records; cancer diagnosis of affected family members was confirmed on pathology reports.

A written informed consent was obtained for blood and tissue sampling before molecular analyses. The study was reviewed and approved by the ethics review board at the University of Sassari.

Mutation screening. For mutation analysis, genomic DNA was isolated from peripheral blood specimens, using standard methods; genes were then screened for mutations using different approaches. All five FA genes (Fanc-A, Fanc-C, Fanc-E, Fanc-F, and Fanc-G) and the two MMR genes (MLH1 and MSH2) were analyzed by denaturing highperformance liquid chromatography (DHPLC) and automated sequencing analysis, according to protocols previously described by our group (13). For Fanc-A gene (including 43 exons), a Multiplex Ligation-dependent Probe Amplification (MLPA) analysis, a highly innovative method that allows a simultaneous identification of large or small deletions and insertions in the coding regions through a multiplex PCR reaction with ligation of the different amplified exons, was also performed. In particular, an MLPA kit specific for Fanc-A gene was used (MRC-Holland, Amsterdam, The Netherlands); the amplification products corresponding to the ligated exons were separated by capillary electrophoresis and peak profiles were visualized using the Genescan software (Applied Biosystems, Foster City, CA). Deletions or insertions in exons are evidenced by a reduction or increase (respectively) of the area under the peak of the sample in comparison to that of the control DNA. Primers sequences corresponding to the 43 exons of Fanc-A, 14 exons of Fanc-C, 10 exons of $F a n c-E$, one exon of $F a n c-F$, and 14 exons of Fanc- $G$ were as reported into the Human Genome DataBase (at: http://www.ncbi.nlm.nih.gov/genome/guide/human). Primer sequences corresponding to the 19 exons of MLH1 and the 16 exons of $M S H 2$ were as previously reported $(24,25)$. All primers and PCR protocols will be also available upon request.

To evaluate the prevalence of each gene variant in a control population, 203 unrelated healthy individuals (corresponding to 406 control chromosomes), originating from the same geographical areas and with no family history for cancer, were used as controls and screened for each sequence variant identified.

Immunohistochemical analysis. Immunohistochemistry (IHC) was performed on $2-\mu \mathrm{m}$ sections of formalin-fixed paraffin-embedded tissues, using standard procedures. Monoclonal antibodies against the candidate proteins were from Santa Cruz Biotechnology Inc. (Santa Cruz, CA, USA). Staining was evaluated semiquantitatively, using normal epithelial cells as internal controls. Intensity and distribution of immunostaining was used to classify tumor samples as positive [strong $(+++)$ to moderate $(++)$ staining, homogeneously distributed or presented by large majority of tumor cells] or negative [absent (-) or weak staining (+)]. The IHC scoring was performed by at least two investigators (in very few borderline cases, classification of immunostaining required additional investigators and was based on the consistency of the magority of them).

\section{Results}

Mutation analysis. A consecutive cohort of 206 patients with diagnosis of colorectal carcinoma was enrolled from March 2006 to June 2008. The series of CRC patients approximately presented the same proportion of males and females [107 $(52 \%)$ and $99(48 \%)$, respectively], with a median age of 52 years (range 33-89). According to the Dukes' stage classification, almost half of the patients had localized disease [Dukes' stages A $(25 ; 12 \%)$ and B $(81 ; 39 \%)$; overall, 106 $(51 \%)]$, with the remaining ones presenting an advanced disease at the time of diagnosis [Dukes' stages C $(70 ; 34 \%)$ and D (30; 15\%); overall, $100(49 \%)]$. Most patients (172/ $206 ; 83 \%$ ) presented a localization of the primary tumour in the distal portions of the large bowels (including sigma and/or rectum). Nineteen (9.2\%) patients had a family history of CRC (at least three affected members among first- and second-degree relatives) 
Table I. Germline mutations in candidate genes.

\begin{tabular}{|c|c|c|c|c|c|c|c|c|c|}
\hline $\begin{array}{l}\text { Positive sporadic } \\
\text { cases }(\%)\end{array}$ & $\begin{array}{c}\text { Positive familial } \\
\text { cases }(\%)\end{array}$ & Gene & Exon & Nucleotide & Codon & $\begin{array}{c}\text { Base } \\
\text { change }\end{array}$ & $\begin{array}{l}\text { Amino acid } \\
\text { change }\end{array}$ & $\begin{array}{l}\text { Mutation } \\
\text { effect }\end{array}$ & $\begin{array}{c}\text { Mutation } \\
\text { designation }\end{array}$ \\
\hline $1(0.5)$ & 0 & Fanc-C & 7 & 800 & 267 & $A \rightarrow G$ & Asn to Ser & Missense & N267S \\
\hline $1(0.5)$ & 0 & Fanc-C & 13 & 1348 & 450 & $\mathrm{G} \rightarrow \mathrm{A}$ & Val to Ser & Missense & V450S \\
\hline $1(0.5)$ & 0 & Fanc-E & 1 & 216 & 11 & $\mathrm{G} \rightarrow \mathrm{A}$ & Ala to $\mathrm{Thr}$ & Missense & A11T \\
\hline $2(1.1)$ & 0 & Fanc-G & 12 & 1538 & 513 & $A \rightarrow G$ & Gln to $\mathrm{Arg}$ & Missense & Q513R \\
\hline 0 & $1(5.3)$ & MLH1 & 8 & 655 & 219 & $A \rightarrow G$ & Ile to Val & Missense & I219V \\
\hline 0 & $1(5.3)$ & MLH1 & 16 & 1851 & 618 & $\mathrm{AA} \rightarrow \mathrm{CG}$ & Lys to Arg & Missense & K618R \\
\hline 0 & $3(15.8)$ & MLH1 & 19 & 2245 & 749 & $\mathrm{~T} \rightarrow \mathrm{C}$ & Leu to Pro & Missense & L749P \\
\hline
\end{tabular}

Description and frequency of identified mutations among 19 familial and 187 sporadic CRC cases are reported.
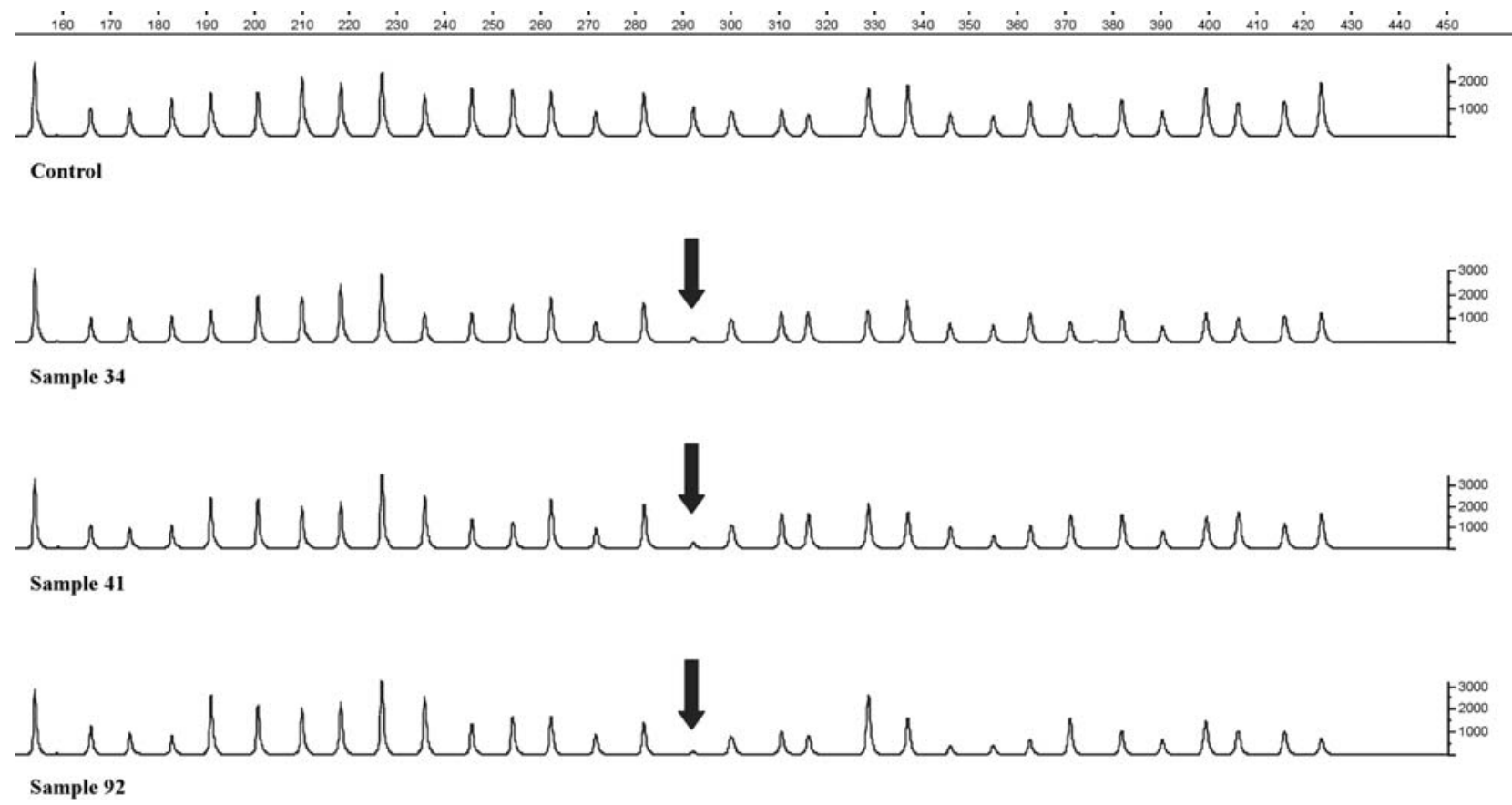

Figure 1. MLPA analysis of Fanc-A gene. Electropherograms are reported for three positive genomic samples from CRC patients of our series, as compared to the normal control from healthy individual. Arrows indicate the reduction of the area under the peak corresponding to the $F a n c-A$ exon 26 .

All CRC patients were firstly evaluated for the occurrence of germline mutations in $M L H 1 / M S H 2$ genes by DHPLC analysis and automated sequencing. Overall, $5(2.4 \%)$ cases presented three different germline variations within the coding sequence of the MLH1 gene: Leu749Pro (3 cases), Lys618Arg, Ile219Val (Table I). All five patients carrying an $M L H 1$ mutation presented a familial recurrence of the disease [in other words, 5/19 (26\%) familial cases presented a germline mutation in $M L H 1$ gene; no $M L H 1 / M S H 2$ germline variant was observed in sporadic CRC cases].

All sequence variants were missense; they were absent in normal genomic DNA from 203 unrelated healthy individuals as controls. While MLH1-I219V and MLH1-K618R should be classified as variants with an unknown functional significance due to the lack of consistent data about the effects on gene products (though MLH1 I219V seems not to directly contribute to the impairment of mismatch repair) (26), the
MLH1-L749P has been indicated as a pathogenic variant by in silico assays (27). In our series, all three CRC cases carrying the MLH1-L749P variant presented a negative staining for the MLH1 protein in primary tumors; a normal MLH1 expression was instead detected for cases carrying the MLH1-I219V and MLH1-K618R variants (see below). Since the patients with the MLH1-L749P variant were not related and such a sequence variation has not been described to be due to the existence of a mutational hot-spot at this genomic level, the recurrence of MLH1-L749P in our population seems to be remarkably coincidental.

Germline mutations in FA genes have been searched using different approaches as described in Materials and methods. An MLPA analysis was carried out for screening gross rearrangements in Fanc-A gene. As shown in Fig. 1, the only alteration of the MLPA profile observed in our series was a reduction of the electrophoretic peak corresponding 

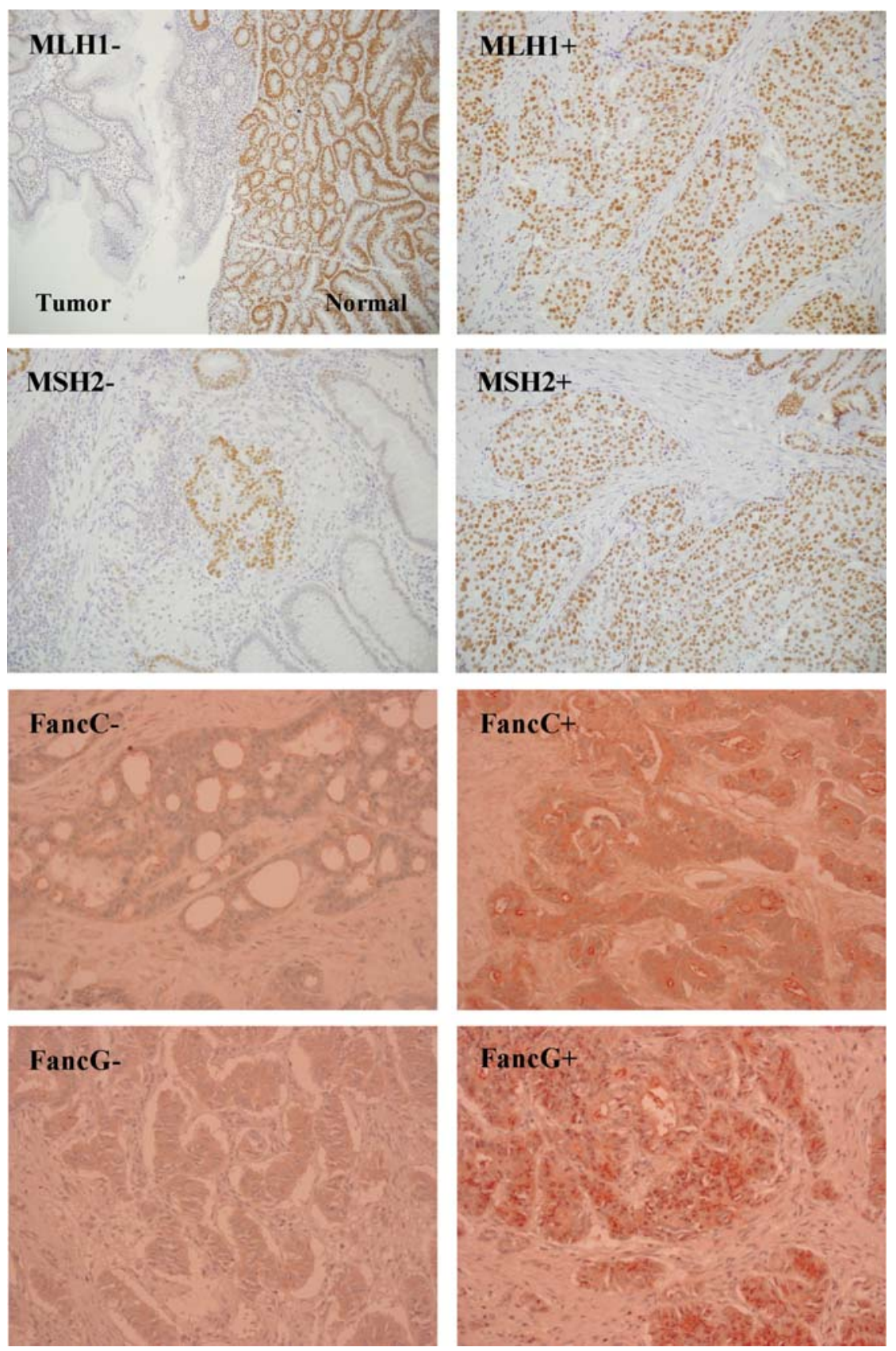

Figure 2. Immunohistochemical staining of candidate proteins. Top, MLH1 and MSH2 expression in primary tumors. Bottom, Fanc-C and Fanc-G expression in hepatic CRC metastases. Images are shown at a magnification of $\mathrm{x} 20$.

to the exon 26 of Fanc-A gene. Such a rearrangement could be classified as a heterozygous deletion since the reduction of the area under the peak was not complete in all mutated samples (such an alteration has not been previously reported). This Fanc-A alteration was observed in 5 (2.4\%) out of 206 sporadic CRC patients. This latter gene as well as the remaining four FA genes (Fanc-C, Fanc-E, Fanc-F, and Fanc-G) were screened for germline mutations in all coding regions and splice boundaries of each gene by DHPLC analysis. PCR products with abnormal denaturing profiles in comparison to the normal controls were sequenced using an automated approach. Two Fanc-C (Asn267Ser and Val450Ser), two Fanc-G (Gln513Arg, 2 cases), and one Fanc-E (Ala11Thr) germline variants were detected in 5/206 $(2.4 \%)$ analysed cases from our series (Table I). All sequence variants were missense mutations and should be classified as genetic alterations with an unknown functional significance due to the lack of any information about the effect on gene proteins. Again, no sequence variation was observed in any of the five FA genes among 203 unrelated healthy individuals (corresponding to 406 control chromosomes).

Expression analysis. We have evaluated the somatic expression of all candidate genes by immunohistochemistry (IHC) on available subsets of tissue samples from primary tumors and liver metastases of CRC patients from our series. Representative examples of IHC staining for MLH1, MSH2 and FA proteins are shown in Fig. 2. Among the 108 primary colorectal carcinomas analyzed in our series, 17 were from familial CRC cases and 91 from sporadic patients. A negative 
Table II. Tissue expression of candidate genes.

\begin{tabular}{|c|c|c|c|c|c|c|c|c|}
\hline Protein & $\begin{array}{l}\text { Analyzed primary } \\
\text { tumors }\end{array}$ & $\begin{array}{c}\text { Familial } \\
\text { cases }(\%) \\
(\mathrm{n}=17)\end{array}$ & $\begin{array}{c}\text { Sporadic } \\
\text { cases }(\%) \\
(\mathrm{n}=91)\end{array}$ & $\begin{array}{c}\text { Negative } \\
\text { staining } \\
\text { cases }\end{array}$ & $\begin{array}{c}\text { Analyzed } \\
\text { metastatic } \\
\text { lesions }\end{array}$ & $\begin{array}{c}\text { Familial } \\
\text { cases }(\%) \\
\quad(n=4)\end{array}$ & $\begin{array}{c}\text { Sporadic } \\
\text { cases }(\%) \\
(\mathrm{n}=35)\end{array}$ & $\begin{array}{c}\text { Negative } \\
\text { staining } \\
\text { cases }\end{array}$ \\
\hline Fanc-C & 108 & 0 & 0 & 0 & 39 & $1(25)$ & $9(26)$ & $10(26)$ \\
\hline Fanc-G & 106 & 0 & 0 & 0 & 39 & $1(25)$ & $8(23)$ & $9(23)$ \\
\hline MLH1 & 107 & $9(53)$ & $16(18)$ & $25(23)$ & 39 & $2(50)$ & $7(20)$ & $9(23)$ \\
\hline MSH2 & 108 & $2(12)$ & $12(13)$ & $14(13)$ & 39 & $1(25)$ & $5(14)$ & $6(15)$ \\
\hline
\end{tabular}

Negative staining results from immunohistochemical analysis on primary and metastatic CRC tissues.

immunostaining for the MLH1 and MSH2 proteins was observed in 25/107 (23\%) and 14/108 (13\%) analyzed patients, respectively (Table II). Among the familial CRC patients, 9/17 (53\%) cases presented loss of the MLH1 protein signal (including the three cases with the MLH1L749P variant) and 2/17 (12\%) cases presented a negative MSH2 protein staining [overall, about two thirds (11/17; $65 \%$ ) of familial CRC patients presented a somatic MMR gene silencing]. On the other hand, lack of MLH1 and MSH2 expression was observed in 16/90 (18\%) and 12/91 (13\%) sporadic CRC cases, respectively [overall, 24/91 (26\%) of these patients showed somatic inactivation of at least one of these two MMR genes, in four cases, a negative staining for both MLH1 and MSH2 proteins was found]. No alteration of the FA gene protein expression was observed in all analyzed primary tumors (Table II).

In a series of 39 available liver CRC metastases, absence of expression was observed in 10 (26\%) samples, for Fanc-C protein, and in $9(23 \%)$ samples, for Fanc-G protein (Table II); no additional FA gene expression was found altered in CRC metastases from the same series. Conversely, a negative MMR staining was observed in metastatic lesions at quite similar rates to those observed in primary tumors: 9/39 (23\%), for MLH1, and 6/39 (15\%), for MSH2 (Table II). A simultaneous lack of expression for MLH1/MSH2 and Fanc-C/-G proteins was observed in $2 / 39(5 \%)$ cases only.

\section{Discussion}

In this study, we performed a mutation analysis of the five main genes (Fanc-A, Fanc-C, Fanc-E, Fanc-F, and Fanc-G) belonging to the Fanconi anemia pathway, which is involved in DNA repair through homologous recombination as well as of the two main genes ( $M L H 1$ and $M S H 2)$ involved in DNA mismatch repair among CRC patients, in order to evaluate the role and relationship of such two pathways during colorectal tumorigenesis.

Absence of any disease-causing mutation in FA genes was observed in our series (with the exception of few sequence variations of unknown functional significance in Fanc-C, Fanc-E, and Fanc-G; Table I). Mutation analysis in MLHI and $M S H 2$ genes revealed an $M L H 1$ mutation in patients with familial recurrence of colorectal cancer (presence of at least three affected family members) only. Considering the MLH1-L749P as the only pathogenic variant in our series, the prevalence of $M L H 1$ mutation in CRC families from our series was very low $(3 / 19 ; 16 \%)$; frequency of mutations in MLH1 and MSH2 genes has been documented at higher rates in different populations, ranging from approximately $25 \%(9,28)$ to more than $80 \%(29,30)$.

When a subset of primay and secondary tumor tissues from different CRC patients were investigated for gene expression by immunohistochemical analysis, a reduced level of Fanc-C and Fanc-G proteins was found in about one fourth of the analyzed liver metastases whereas a normal expression of all FA proteins was observed in the analyzed primary tumors. On the other hand, low staining signals for MLH1 and MSH2 proteins were found at a quite identical prevalence between primary and secondary CRC lesions; altogether, about one third $(35 / 108 ; 32 \%)$ of analyzed cases presented low expression of such gene products) (Table II). Based on these results, one could speculate that: i) Fanc-C and $F a n c-G$ genes may be silenced during disease progression and metastasis formation; and ii) inactivation of $\mathrm{MLH1}$ and $\mathrm{MSH} 2$ genes may occur before tumor disse-mination and maintained at the same level during tumor progression.

Although the number of cases should be increased, our findings seem to indicate that functional alterations in mismatch repair and FA pathways may represent two different pathogenetic mechanisms which are concurring to colorectal cancer progression. While deficit in mismatch DNA repair may induce replication errors and genetic instability with accumulation of further mutations in target cancer genes (3), alterations of the FA pathway may affect the molecular machinery that monitors the proper progression of the cell cycle $(31,32)$. In this regard, the FA pathway may have a specific role in repairing DNA crosslinking damage through coordination of at least three DNA repair pathways: homologous recombination, non-homologous endjoining, and translesion DNA synthesis (32). Consequently, inactivation of any of the FA genes may lead to chromosome instability and karyotypic anomalies (31). Indeed, in colorectal cancer, an inverse relationship between genetic instability and karyotypic alterations has been described (cancers showing genome-wide microsatellite instability are, in general, diploid and exhibit normal rates of gross chromosomal changes, whereas stable tumors are usually 
aneuploid and exhibit increased rates of chromosomal changes) $(6,7)$.

Further experiments on larger collections of different types of primary and secondary tumor tissues are needed to further assess whether functional alterations of the FA genes may cooperate with known molecular events in colorectal tumorigenesis.

\section{Acknowledgements}

Authors are grateful to patients for their important contribution to this study. The study was supported by Italian Ministry of Health 'Progetto Ricerca Finalizzata', Amgen Inc., and Sardinia Regional Government (Regione Autonoma della Sardegna).

\section{References}

1. Curado MP, Edwards B, Shin HR, Storm H, Ferlay J, Heanue M and Boyle P (eds). Cancer Incidence in Five Continents. Vol. IX. International Agency for Research on Cancer (IARC) Scientific Publications, IARC, Lyon, No. 160, 2007.

2. Walther A, Johnstone E, Swanton C, Midgley R, Tomlinson I and Kerr D: Genetic prognostic and predictive markers in colorectal cancer. Nat Rev Cancer 9: 489-499, 2009.

3. Grady WM and Markowitz S: Genomic instability and colorectal cancer. Curr Opin Gastroenterol 16: 62-67, 2000.

4. Ishiguro $K$, Yoshida T, Yagishita $H$, Numata $Y$ and Okayasu $T$ : Epithelial and stromal genetic instability contributes to genesis of colorectal adenomas. Gut 55: 695-702, 2006.

5. Olschwang S, Hamelin R and Laurent-Puig P: Alternative genetic pathways in colorectal carcinogenesis. Proc Nat Acad Sci USA 94: 12122-12127, 1997.

6. Lengauer C, Kinzier KW and Volgestein B: Genetic instabilities in human cancers. Nature 396: 643-649, 1998.

7. Vogelstein B, Fearon ER and Hamilton SR: Genetic alterations during colorectal-tumor development. N Engl J Med 319: 525-532, 1998

8. Gonzalez-Garcia I, Moreno V and Navarro M: Standardized approach for microsatellite instability detection in colorectal carcinomas. J Natl Cancer Inst 92: 544-549, 2000.

9. Caldes T, Godino J and de la Hoya M: Prevalence of germline mutations of MLH1 and MSH2 in hereditary non-polyposis colorectal cancer families from Spain. Int J Cancer 98: 774-779, 2002.

10. Cederquist K, Emanuelsson $\mathrm{M}$ and Goransson I: Mutation analysis of the MLH1, MSH2 and MSH6 genes in patients with double primary cancers of the colorectum and the endometrium: a population-based study in northern Sweden. Int J Cancer 109: 370-376, 2004.

11. Peltomaki P: Deficient DNA mismatch repair: a common etiologic factor for colon cancer. Hum Mol Genet 10: 735-740, 2001.

12. Strazzullo M, Cossu A, Baldinu P, Colombino M, Satta M, Tanda F, De Bonis M, Cerase A, D'Urso M, D'Esposito M and Palmieri G: High-resolution methylation analysis of hMLH1 promoter in sporadic endometrial and colorectal carcinomas. Cancer 98: 1540-1546, 2003.

13. Colombino M, Cossu A, Budroni M, Satta MP, Baldinu P, Casula M, Palomba G, Pisano M, Sini MC, Deiana A, Tanda F and Palmieri G: Identification of predictive factors for the occurrence of predisposing MLH1 and MSH2 germline mutations among Sardinian patients with colorectal carcinoma. Eur J Cancer 41: 1058-1064, 2005.
14. D'Andrea AD: Fanconi anemia. Curr Biol 13: 546, 2003.

15. D'Andrea AD: Susceptibility pathways in Fanconi's anemia and breast cancer. N Engl J Med 362: 1909-1919, 2010.

16. Medhurst AL, Huber PA and Waisfisz Q: Direct interactions of the five known Fanconi anaemia proteins suggest a common functional pathway. Hum Mol Genet 10: 423-429, 2001.

17. Garcia-Higuera I, Taniguchi $\mathrm{T}$ and Ganesan S: Interaction of the Fanconi anemia proteins and BRCA1 in a common pathway. Mol Cell 7: 249-262, 2001.

18. Zhang F, Ma J, Wu J, et al: PALB2 links BRCA1 and BRCA2 in the DNA-damage response. Curr Biol 19: 524-529, 2009.

19. Sy SM, Huen MS and Chen J: PALB2 is an integral component of the BRCA complex required for homologous recombination repair. Proc Natl Acad Sci USA 106: 7155-7160, 2009.

20. Chen CC, Taniguchi T and D'Andrea A: The Fanconi anemia (FA) pathway confers glioma resistance to DNA alkylating agents. J Mol Med 85: 497-509, 2007.

21. Farmer $\mathrm{H}, \mathrm{McCabe} \mathrm{N}$, Lord $\mathrm{CJ}$, et al: Targeting the DNA repair defect in BRCA mutant cells as a therapeutic strategy. Nature 434: 917-921, 2005.

22. Fong PC, Boss DS, Yap TA, et al: Inhibition of poly(ADPribose) polymerase in tumors from BRCA mutation carriers. N Engl J Med 361: 123-134, 2009.

23. Cunningham D, Atkin W, Lenz HJ, Lynch HT, Minsky B, Nordlinger B and Starling N: Colorectal cancer. Lancet 375: 1030-1047, 2010.

24. Baldinu P, Cossu A, Manca A, Satta MP, Pisano M, Casula M, Dessole S, Pintus A, Tanda F and Palmieri G: Microsatellite instability and mutation analysis of candidate genes in unselected Sardinian patients with endometrial cancer. Cancer 94: 3157-3168, 2002.

25. Colombino M, Cossu A, Arba A, Manca A, Curci A, Avallone A, Comella G, Botti G, Scintu F, Amoruso M, D'Abbicco D, d'Agnessa MR, Spanu A, Tanda F and Palmieri G: Microsatellite instability and mutation analysis among Southern Italian patients with colorectal carcinoma: detection of different alterations accounting for MLH1 and MSH2 inactivation in familial cases. Ann Oncol 14: 1530-1536, 2003.

26. Plotz G, Raedle J, Spina A, Welsch C, Stallmach A, Zeuzem S and Schmidt C: Evaluation of the MLH1 I219V alteration in DNA mismatch repair activity and ulcerative colitis. Inflamm Bowel Dis 14: 605-611, 2008.

27. Gerykova-Bujalkova M, Krivulcik T and Bartosova Z: Novel approaches in evaluation of pathogenicity of single-base exonic germline changes involving the mismatch repair genes MLH1 and MSH2 in diagnostics of Lynch syndrome. Neoplasma 55: 463-471, 2008.

28. Katballe N, Christensen M, Wikman FP, Orntoft TF and Laurberg S: Frequency of hereditary non-polyposis colorectal cancer in Danish colorectal cancer patients. Gut 50: 43-51, 2002.

29. Wang Q, Lasset C and Desseigne F: Prevalence of germline mutations of hMLH1, hMSH2, hPMS1, hPMS2, and hMSH6 genes in 75 French kindreds with non-polyposis colorectal cancer. Hum Genet 105: 79-85, 1999.

30. Wagner A, Barrows A and Wijnen JT: Molecular analysis of hereditary non-polyposis colorectal cancer in the United States: high mutation detection rate among clinically selected families and characterization of an American founder genomic deletion of the MSH2 gene. Am J Hum Genet 72: 1088-1100, 2003.

31. Kennedy RD, and D'Andrea AD: DNA Repair pathways in clinical practice: lessons from pediatric cancer susceptibility syndromes. J Clin Oncol 24: 3799-3808, 2006.

32. Mathew CG: Fanconi anaemia genes and susceptibility to cancer. Oncogene 25: 5875-5884, 2006. 